\title{
Development of a circular polarized fractal antenna
}

\author{
Alexandr Savochkin ${ }^{1, *}$ and Alexander Nudga $^{2}$ \\ ${ }^{1}$ Sevastopol State University, st. Universitetskaya, 33, 299053, Sevastopol, Russia \\ ${ }^{2}$ Crimean Federal University named V.I. Vernadsky, Vernadsky, 4, 295007, Simferopol, Russia
}

\begin{abstract}
The article presents the results of analytical work, computer modeling, and experimental studies of the fractal antenna of circular polarization. The antenna is a flat structure parallel to the screen. The phase shifts necessary for the operation of the antenna are provided by delay lines. The antenna operates in the frequency range of $902 \ldots 928 \mathrm{MHz}$, an experimental study of its characteristics was performed in the same range.
\end{abstract}

\section{Introduction}

In recent years, success has been achieved in the research and development of new types of antennas. One of these types are fractal antennas. Their feature is self-similarity, which allows you to work simultaneously in several frequency ranges [1]. This fact is of great practical interest. Currently, most structures are analyzed by calculation and experimental methods, which provides a wide field for research and the search for a generalized physical approach for the development of new structures [2, 3, 4].

This paper presents the results of applying the calculation and experimental method for determining the characteristics of the antenna: radiation pattern (radiation pattern), standing wave ratio (SWR).

\subsection{Analytical part}

\subsubsection{Purpose}

The aim of the work is to create a new flat circular polarized antenna that can provide operation at several frequency ranges. The developed antenna is designed to be used either as a standalone antenna or as an element of the antenna array.

\subsubsection{Current state}

Circular polarization antennas are known that can emit a circular polarization field when two degenerate orthogonal modes of oscillation with a relative phase shift of 90 degrees are excited in them. Such antennas use flat emitters of usually round or square shape with the

*Corresponding author: savochkin_mail@mail.ru 
inclusion of two orthogonally located exciters, providing a phase shift of 90 degrees. The emitter is excited from a common power line and divider by additional line segments providing the necessary phase shift [1]. These antennas have a disadvantage - the inability to work in several frequency ranges, since the emitter forms a circular polarization field in only one frequency range.

The closest in technical essence to the developed antenna is a multi-band antenna, consisting of a screen and a flat emitter of a triangular shape, perpendicular to the plane of the screen. The power point for this antenna is connected to one of the vertices of a flat emitter of a triangular shape, which is located closest to the screen [2]. The triangular flat emitter is made in the form of a fractal structure based on the Sierpinski napkin.

Fractal structures of this type correspond to the principle of self-similarity and do not have a characteristic size, since an infinite number of scales and sizes are observed in one object. Due to this property, fractal structures are multiband in the electromagnetic sense.

These antennas have a disadvantage - the impossibility of creating a circular polarization field, since antennas made on the basis of the Sierpinski napkin form a linear polarization field. The vector of the electric field $\mathrm{E}$ for such antennas is oriented perpendicular to the plane of the antenna screen, and the vector of the magnetic field $\mathrm{H}$ is oriented parallel to the plane of the screen.

Using a linear polarization field is not advisable if the antennas are located on move mobile devices. To avoid possible problems of reception and transmission of radio waves, circular polarized antennas should be used.

The antenna structure includes a flat emitter of a triangular shape and a metal screen (Fig. 1), while a flat emitter of a triangular shape is made with resonant holes of a triangular shape. The flat emitter of a triangular shape is parallel to the plane of the screen and is at a distance $\mathrm{d}$ selected from the relation

$$
0,005 \lambda \leq \mathrm{d} \leq 0,15 \lambda
$$

where $\lambda-$ is the average wavelength.

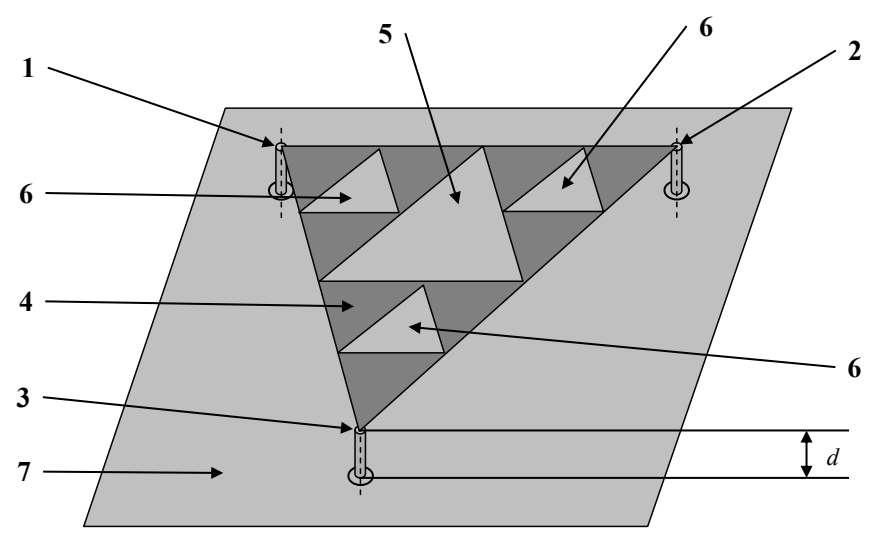

Fig. 1. Multi-band circular polarized antenna (1,2,3 - the vertices of a flat emitter of a triangular shape, 4 - a flat emitter of a triangular shape, 5, 6- resonant holes of a triangular shape, 7 - metal screen)

A flat triangular emitter is excited at the vertices by currents of equal amplitude with phases 0, 120, 240 degrees for the first, second, and third vertices, respectively.

The location of the flat emitter of a triangular shape of the antenna above a parallel screen, as well as the excitation of a flat emitter of a triangular shape by currents at three vertices with a given phase shift allows you to create a circular polarization field.

The advantage of this multi-band circular polarized antenna is the relative ease of implementation and compactness of the planar structure. All this allows you to use this type of antenna for operation in several frequency ranges. 
A multi-band circular polarized antenna, the circuit of which is shown in Fig. 1, consists of a flat emitter of a triangular shape 4 with resonant holes of a triangular shape 5, 6 and a metal screen 7 . The choice of distance $d$ is based on the condition of a single-mode excitation of an emitter of a triangular shape.

A multi-band antenna circular polarized in the mode of, for example, radiation at a frequency of one of the operating ranges operates as follows. The top 1 of a triangular flat emitter is excited by a current with phase $0^{\circ}$, which creates electromagnetic waves in the structure without an additional phase shift $\left(0^{\circ}\right)$; peak 2 of a triangular flat emitter is excited by a current with a phase of $120^{\circ}$, which creates electromagnetic waves in the structure with a phase shift of $120^{\circ}$ (relative to the electromagnetic waves generated by the excitation of peak 1); peak 3 of a triangular flat emitter is excited by a current with a phase of $240^{\circ}$, which creates electromagnetic waves in the structure with a phase shift of $240^{\circ}$ (relative to the electromagnetic waves generated by excitation of peak 1). In this case, the total electromagnetic field of the antenna in the radiation mode will have circular polarization, since the partial field vectors excited by different vertices are also shifted $120^{\circ}$ relative to each other in space.

As a result of the superposition of the fields created by the antenna upon excitation of a triangular flat emitter at individual vertices, a circular polarization field is formed on an axis perpendicular to the plane of a triangular flat emitter and passing through its center.

Moreover, the non-uniformity of the field formed by the antenna when using three excitement points at three vertices will be less than when using analogs of the antenna with two orthogonally located excitement providing a phase shift of $90^{\circ}$.

This multi-band circular polarization antenna can be used instead of several single-band circular polarization antennas.

Thus, this design of a multi-band antenna exhibits new consumer properties: reducing the thickness of the antenna and the possibility of forming a circular polarization field in several frequency ranges, which allows to improve the operation of communication systems when using circular polarized antennas.

\section{Modeling and experiment part}

Process modeling was carried out in the FEKO electrodynamic analysis package. For frequency band $902 \ldots 928 \mathrm{MHz}$, the radiation pattern was measured at frequencies of 902 $\mathrm{MHz}, 915 \mathrm{MHz}, 928 \mathrm{MHz}$ for two orthogonal orthogonal sections. The following instruments were used to conduct experimental studies of the radiation characteristics: a G4-79 generator, an auxiliary P6-23A horn antenna with aperture dimensions of $0.342 \times 0.256 \mathrm{~m}$, and a receiving device - a Spectran HF-6085 spectrum analyzer manufactured by Aaronia $A G$. The input characteristics of the antenna were determined in the frequency band from 850 to $950 \mathrm{MHz}$ using the Anritsu Site Master S331A instrument.

\subsection{Settlement and experimental determination method RP and SWR}

To determine the radiation pattern and SWR, the calculation-experimental method was used. A triangular circular polarization antenna, which is a first-order fractal, was investigated. The results of the simulation performed in the FEKO package are shown in Fig. 2. For an experimental study, a sample was made; the photo is shown in Fig. 3. Layout for printing microstrip phase shift lines feeding the antenna shown in Fig.4. To measure the $\mathrm{RP}$, the method for determining the field in the far zone was selected, in Fig. 5. shows the 
obtained pattern at the center frequency of the range. The frequency dependence of the SWR is shown in Fig. 6.

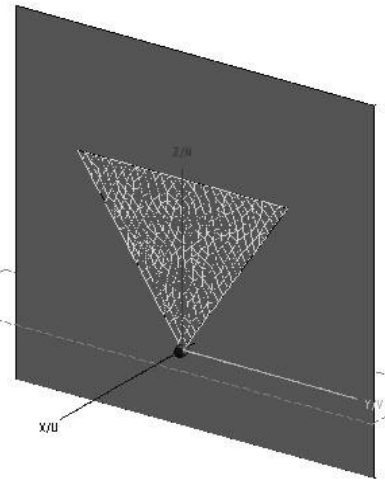

Fig. 2. Antenna model in package $F E K O$.

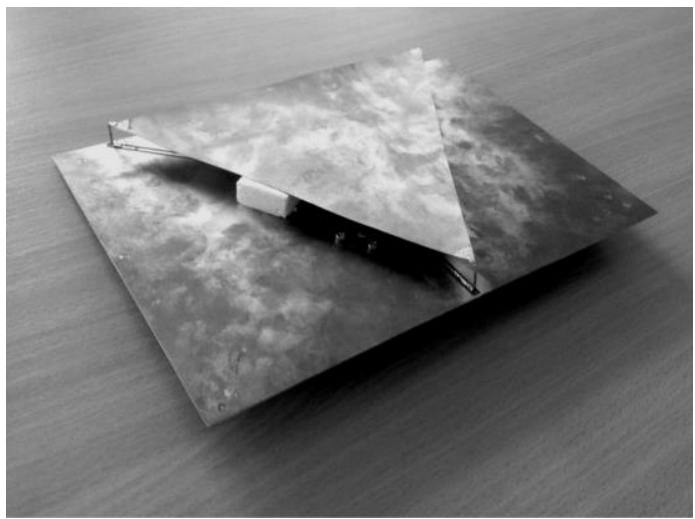

Fig. 3. Sample of the manufactured antenna.

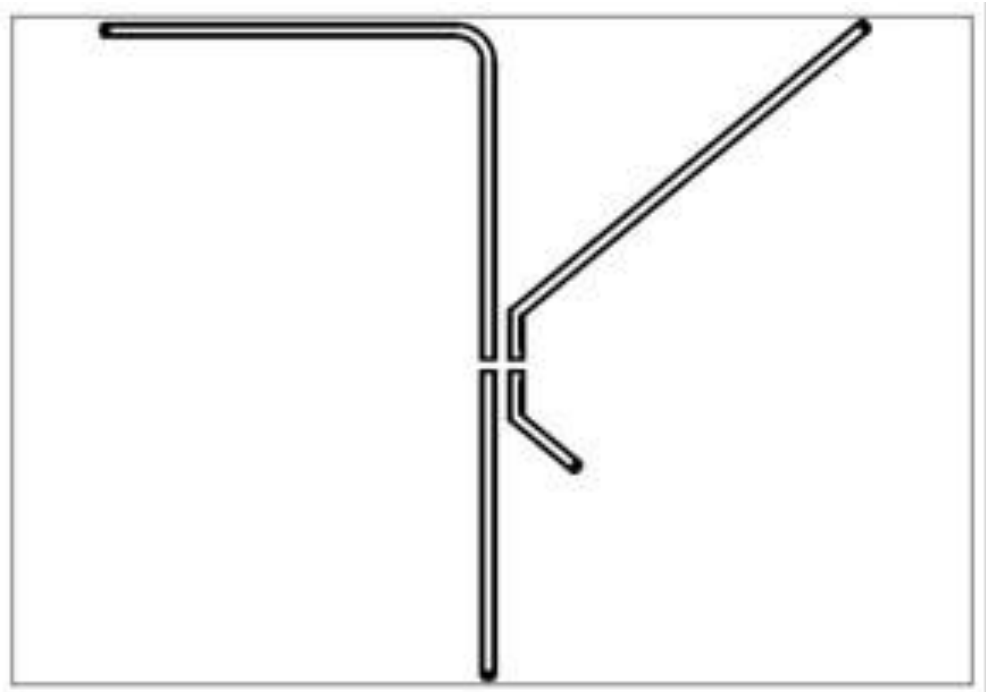

Fig. 4. Layout for printing microstrip phase shift lines feeding the antenna.

$$
F(\theta), \mathrm{dB}
$$

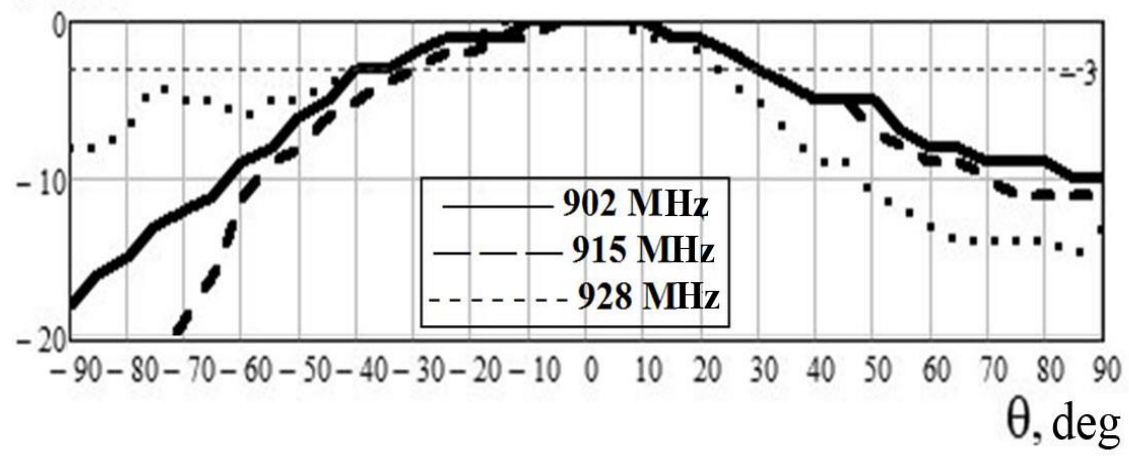

Fig. 5. Antenna patterns. 


\section{SWR}

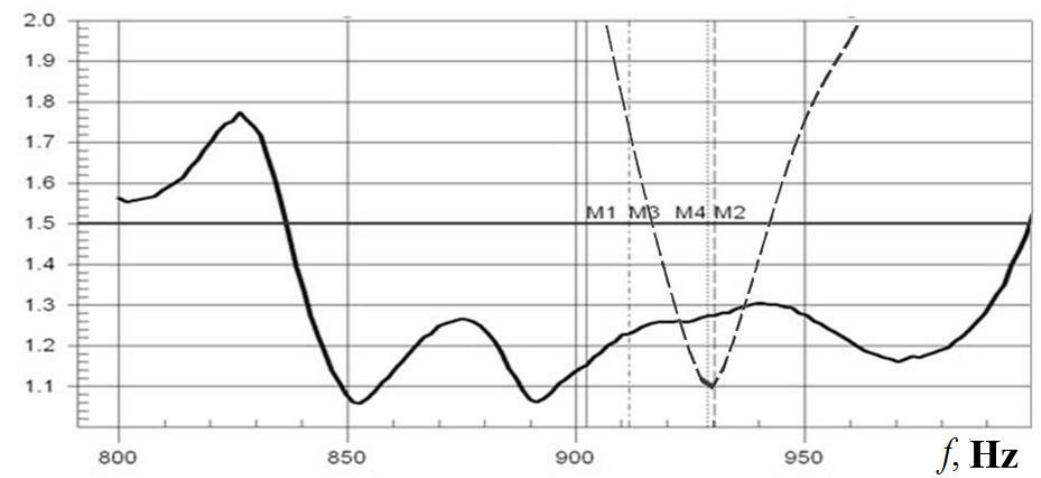

Fig. 6. SWR of antenna.

The measurement results show that the circular polarized antenna forms the main lobe of the axisymmetric shape. The SWR level for the antenna input in the frequency band of interest does not exceed 1.3

\section{Conclusion}

The calculation of the characteristics of a triangular circular polarized antenna is carried out. The level of coordination in the entire band of frequencies of interest indicates the practical application of the test sample and makes it possible to implement fractal antennas of the following orders, which will make it possible to use the antenna simultaneously in several frequency ranges. Upgrading to a multi-band antenna will entail the use of a phase shifter, which in turn will complicate the design and may partly degrade the performance of the system, but cannot change the physics of the process and the organization of the multiband operation mode.

The analytical and experimental characteristics obtained allow us to talk about the possibility of using the antenna in telecommunication devices of mobile communication systems, radio frequency identification systems.

\section{References}

1. D.H. Werner An Overview of Fractal Antenna Engineering Research, IEEE Ant. and Propag. Mag., 45(1). pp. 38-57 (2003)

2. G.F. Tsachtsiris Analysis of a Modified Sierpinski Gasket Monopole Antenna Printed on Dual Band Wireless Devices, IEEE Trans. on Ant. and Propag. 52(10). pp. 25712579 (2004)

3. A.A. Nudga, A.A. Savochkin Triangular antenna of linear polarization, International scientific and technical conference Radio engineering fields, signals, devices and systems (theory, practice, history, education), pp. 103-104 (2013)

4. A.A. Nudga Calculation method of radiation multi-band antennas in the far field, Ultrawideband and Ultrashort Impulse Signals «UWBUSIS-12»: 6-th International conference, pp. 189-191 (2012) 